\title{
CT and PET-CT of a Dog with Multiple Pulmonary Adenocarcinoma
}

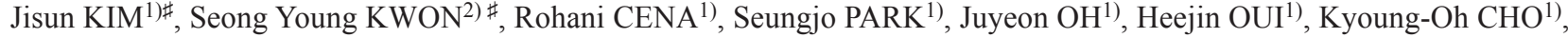 \\ Jung-Joon $\mathrm{MIN}^{2)}$ and Jihye $\mathrm{CHOI}^{1) *}$ \\ ${ }^{1)}$ College of Veterinary Medicine, Chonnam National University, Gwangju 500-757, Korea \\ ${ }^{2)}$ Department of Nuclear Medicine, Chonnam National University Hwasun Hospital, Jeonnam 519-763, Korea
}

(Received 28 August 2013/Accepted 21 December 2013/Published online in J-STAGE 31 December 2013)

ABSTRACT. A 10-year-old, intact female Yorkshire terrier had multiple pulmonary nodules on thoracic radiography and ultrasonography with no lesions elsewhere. Computed tomography (CT) and positron emission tomography and computed tomography (PET-CT) using $18 \mathrm{~F}$-fluorodeoxyglucose (FDG) were performed to identify metastasis and undetected primary tumors. On CT examination, pulmonary nodules had a hypoattenuating center with thin peripheral enhancement, suggesting ischemic or necrotizing lesion. In PET-CT at 47 min after intravenous injection of $11.1 \mathrm{MBq} / \mathrm{kg}$ of $\mathrm{FDG}$, the maximum standardized uptake value of each pulmonary nodule was about from 3.8 to 6.4. There were no abnormal lesions except for four pulmonary nodules on the CT and PET-CT. Primary lung tumor was tentatively diagnosed, and palliative therapy using $2 \mathrm{mg} / \mathrm{kg}$ tramadol and $2.2 \mathrm{mg} / \mathrm{kg}$ carprofen twice per day was applied. After the dog's euthanasia due to deteriorated clinical signs and poor prognosis, undifferentiated pulmonary adenocarcinoma was diagnosed through histopathologic and immunochemistry examination. To the best of the authors' knowledge, this is the first study of CT and PET-CT features of canine pulmonary adenocarcinoma. In this case, multiple pulmonary adenocarcinoma could be determined on the basis of FDG PET-CT through screening the obvious distant metastasis and/or lymph node invasions and excluding unknown primary tumors.

KEY WORDS: computed tomography, fluorodeoxyglucose, multiple pulmonary nodules, positron emission tomography, pulmonary adenocarcinoma.

doi: 10.1292/jvms.13-0434; J. Vet. Med. Sci. 76(4): 615-620, 2014

The etiology of lung masses, including nodules (spots on the lung that are $3 \mathrm{~cm}$ in diameter or less), can be assumed on the basis of morphological characteristics, and especially, the number of masses can act as an important clue for differential diagnosis. Solitary masses may be primary lung tumor, abscess, granuloma or hematoma, whereas multiple masses may be metastatic tumors, septic emboli or parasitic granulomas [19]. However, primary lung tumor can metastasize to lymph nodes, distant organs or to other lung regions through hematogeneous or lymphatic routes or it can spread to adjacent lung tissues through local invasion $[2,3]$. Therefore, primary lung tumor is not necessarily presented as a solitary mass, but may also present itself as multiple masses or as in a disseminated form $[4,14,15]$. Surgical excision is the primary option for primary lung tumor, regardless of whether solitary or multiple types are present and the goal of the surgery is to remove all the gross lesions. Therefore, the primary lung tumor with multiple masses should be differentiated with metastatic lung tumors. When multiple masses are presented, the largest one is usually considered to be the primary lesion. However, if the masses' sizes are equal, it is impossible to determine which one is the origin [13]. Diagnostic imaging with high anatomic resolution, such

*Correspondence to: Chol, J., College of Veterinary Medicine, Chonnam National University, Yongbong-ro, Buk-gu, Gwangju 500-757, South Korea. e-mail: imsono@chonnam.ac.kr

(C2014 The Japanese Society of Veterinary Science

This is an open-access article distributed under the terms of the Creative Commons Attribution Non-Commercial No Derivatives (by-nc-nd) License $<$ http://creativecommons.org/licenses/by-nc-nd/3.0/>. as computed tomography $(\mathrm{CT})$, can be applied to evaluate the tumors in more detail to determine the treatment plan and prognostic factors through estimation of the tumor size, lymph node involvement and pulmonary or distant metastasis [14]. Positron emission tomography (PET), a functional imaging modality, can be used to determine the tumor or metastasis in equivocal situations in which enlarged lesions or those which have altered their shape due to metastasis, are not identified with anatomic imaging during early stages or according to tumor type [18]. PET-CT provides the combined trans-sectional images which consist of functional information of PET superimposed on anatomic information obtained via CT scanning. PET-CT is considered to be one of the most sensitive diagnostic modalities for evaluating metastasis, tumor staging and responses to therapy [9]. In veterinary medicine, the physiologic values of $18 \mathrm{~F}$-fluorodeoxyglucose (FDG) uptake in normal dogs and a few cancer cases studied via PET-CT were reported, in spite of the limited availability and cost of this modality $[2,7,10,11]$. In this study, we described the application of CT and PET-CT to distinguish primary lung tumor from metastatic lung tumor in a dog presenting multiple lung masses.

A 10-year-old, intact female Yorkshire terrier weighing $3.3 \mathrm{~kg}$ was presented at the Chonnam National University Veterinary Teaching Hospital for a general check-up without any specific clinical signs. Physical examination revealed small sized tumors (about $3 \mathrm{~mm}$ diameter) at the left $2 \mathrm{nd}$ and 4th mammary glands. Complete blood count revealed leukocytosis $(40.14 \mathrm{~K} / \mu l$; reference range 5.05-16.76 K/ $\mu l)$. There was no abnormal finding in serum chemistry.

Thoracic radiographs revealed a total of four nodules on 
A

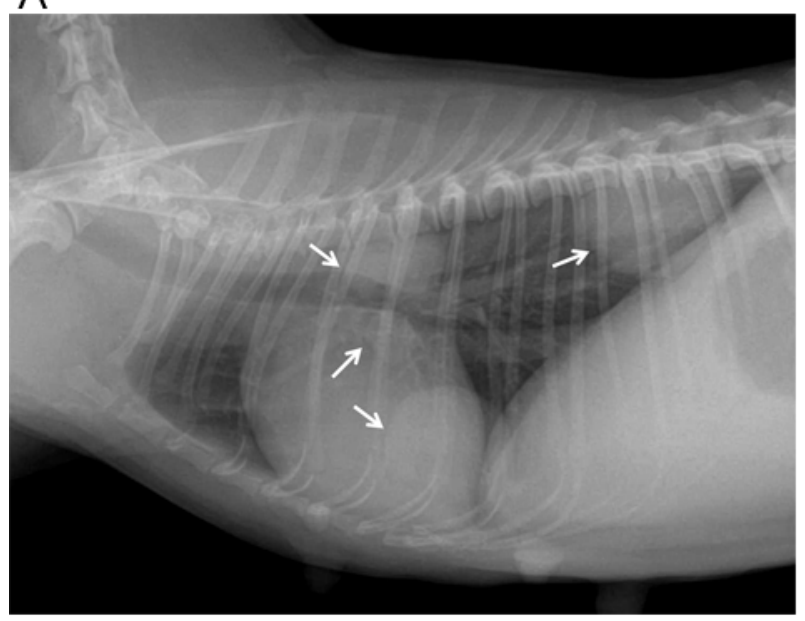

B

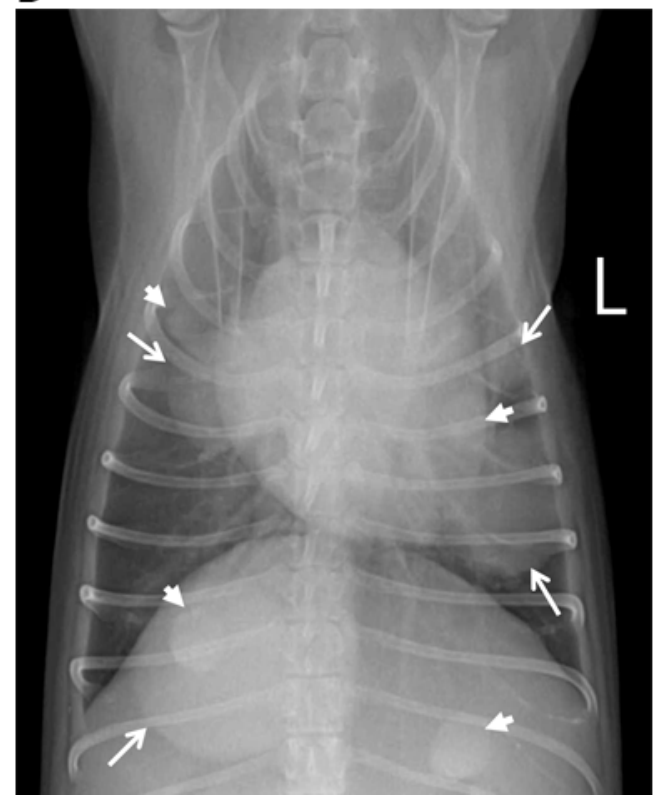

Fig. 1. Canine thoracic radiographs (right lateral and ventrodorsal views), showing four nodules (long arrows) with uniform soft tissue density on the left and right cranial and caudal lobes. Enlarged teats (short arrows) were superimposed on the radiographs.

the left and right cranial and caudal lobes (Fig. 1). All nodules had soft tissue density with definite contour. The largest one, located at the right caudal lobe, was $4 \mathrm{~cm}$ in diameter, and the others were about $2-3 \mathrm{~cm}$. On ultrasonography, all pulmonary nodules had slightly heterogeneous internal hypoechotexture confined by an echogenic border. The blood flow signal was not identified within all nodules on color Doppler mode. There was no remarkable finding on abdominal radiography and ultrasonography. Multiple pulmonary nodules were suspected as metastatic lesions, however, the primary tumor was not identified. Thus, computed tomography (CT) and positron emission tomography-computed tomography (PET-CT) were performed under general anesthesia of a combination of $2.5 \mathrm{mg} / \mathrm{kg}$ zolazepam/tiletamine (Zoletil, Virbac, France) and $0.05 \mathrm{mg} / \mathrm{kg}$ medetomidine (Domitor, Orion Corp., Espoo, Finland) to identify the undetected primary tumor and additional metastasis which may have been present in other organs. Upon CT examination, all lung nodules showed a similar appearance with heterogeneous density consisting of a hypoattenuating center (Fig. 2). The mean density of lung nodules was $27.28 \pm 6.19 \mathrm{HU}$. The size of each nodule was $2.2 \times 2.0 \mathrm{~cm}$ (right cranial nodule), $3.2 \times 2.7 \mathrm{~cm}$ (right caudal nodule), $1.0 \times 1.0 \mathrm{~cm}$ (left cranial nodule) and $2.7 \times 2.0 \mathrm{~cm}$ (left caudal nodule), respectively. Only the peripheral parenchyma of nodules was partially enhanced after an iodine contrast injection (iohexol, Omnihexol 300, Korea United Pharm Co., Seoul, Korea). Thus, most of the parenchyma was considered to have undergone an ischemic or necrotic lesion. PET-CT (Discovery 600 PET/CT system, GE Healthcare, Wauwatosa, WI, U.S.A.) using FDG was performed at $47 \mathrm{~min}$ after the administration of $11.1 \mathrm{MBq} / \mathrm{kg}$ of FDG intravenously. The increased FDG uptake was observed from the peripheral region of each nodule and the maximum standardized uptake values were as follows; 6.4 of the right cranial nodule, 4.3 of the right caudal nodule, 3.8 of the left cranial nodule and 4.7 of the left caudal nodule, respectively (Figs. 3 and 4). There was no evidence of FDG uptake from other regions, including the mammary gland nodules. None other than four pulmonary nodules were detected on CT and PET-CT. Ultrasound guided tru-cut biopsy was performed for the right and left caudal lung nodules. However, histopathological diagnosis was not possible due to extensive necrosis of the sample. Multiple lung nodules in the dog were tentatively diagnosed as a primary lung tumor, because there was no evidence of an unknown primary tumor on the basis of the diagnostic imaging. Complete surgical excision was impossible due to the nodules' diverse sites. Palliative treatment using $2 \mathrm{mg} /$ kg tramadol (tramadol, Dongkwang Pharm, Seoul, Korea) and $2.2 \mathrm{mg} / \mathrm{kg}$ carprofen (rimadyl, Pfizer, New York, NY, U.S.A.) was applied to the dog twice per day for 3 months. Finally, after respiratory distress began to emerge, the owner requested euthanasia of the dog in view of the poor prognosis, and necropsy was performed. Histologically and immunochemically, the lung nodules were diagnosed as undifferentiated pulmonary adenocarcinoma with extensive necrosis, and there was no other tumor identified anywhere in the body, including the mammary gland.

This case had multiple pulmonary adenocarcinoma. Several studies evaluated the number of lung masses in dogs 
A

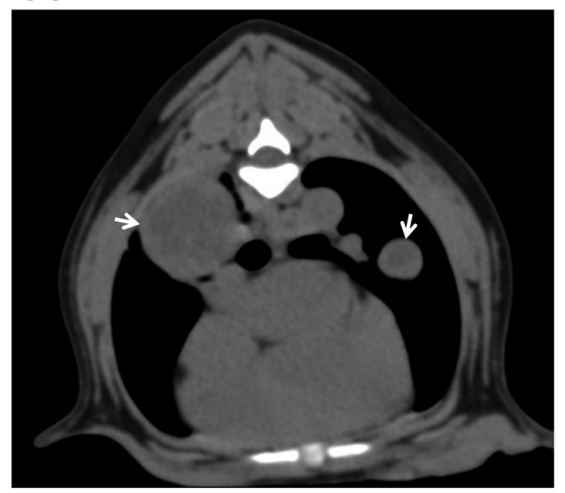

$\mathrm{D}$

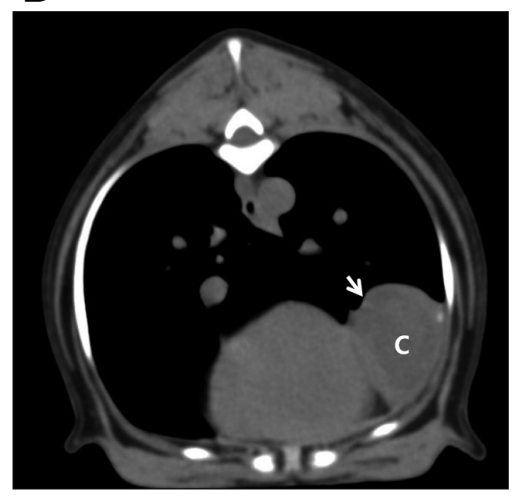

G

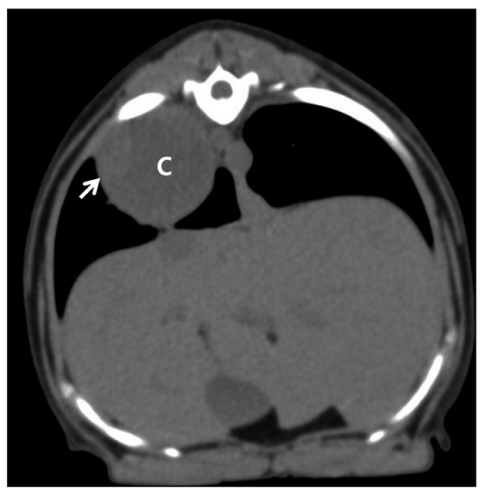

$J$

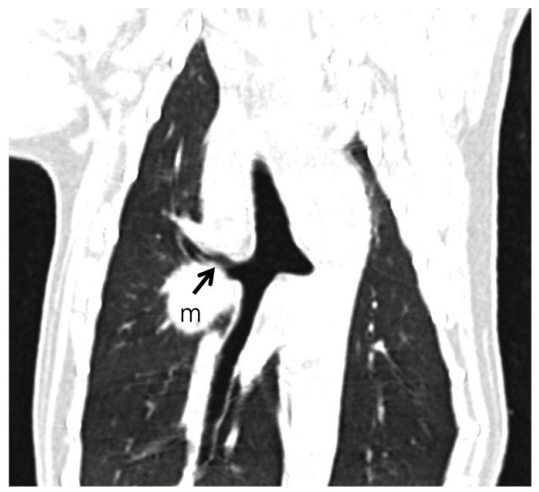

B

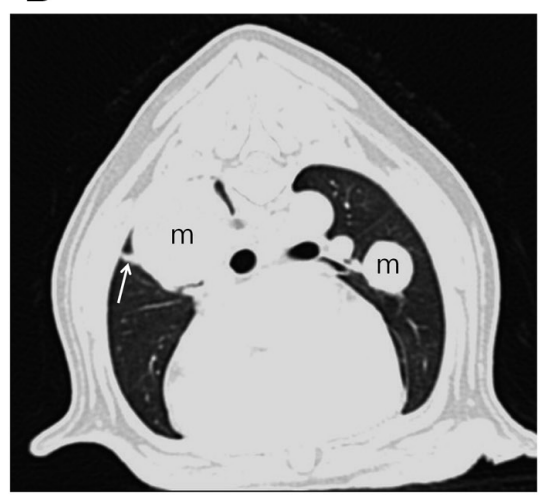

E

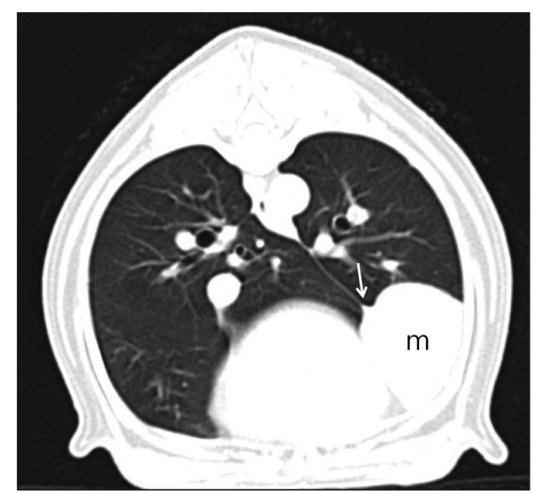

$\mathrm{H}$

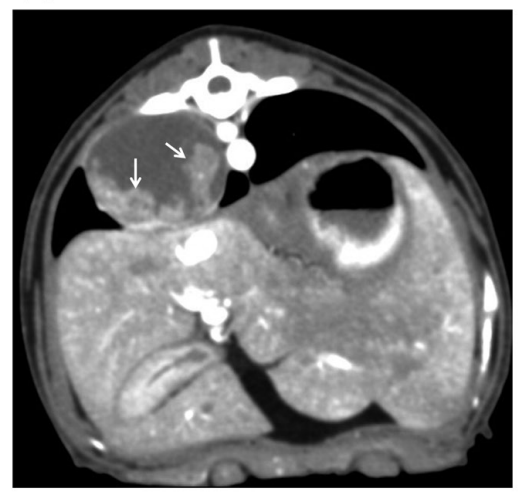

C

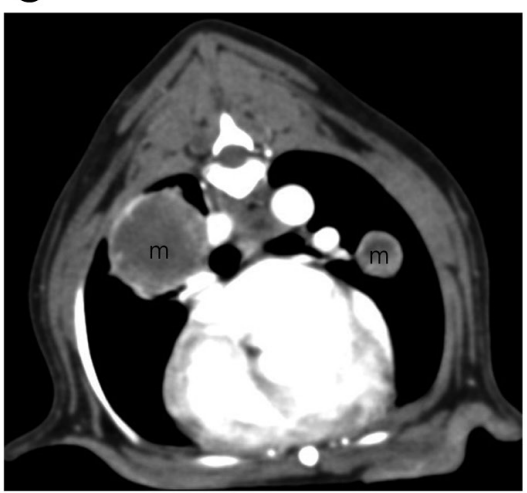

$\mathrm{F}$

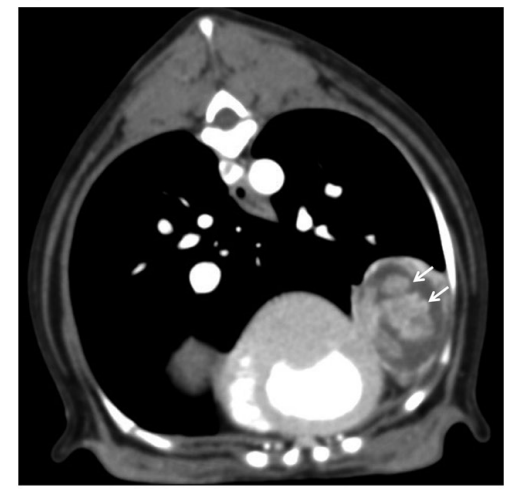

।

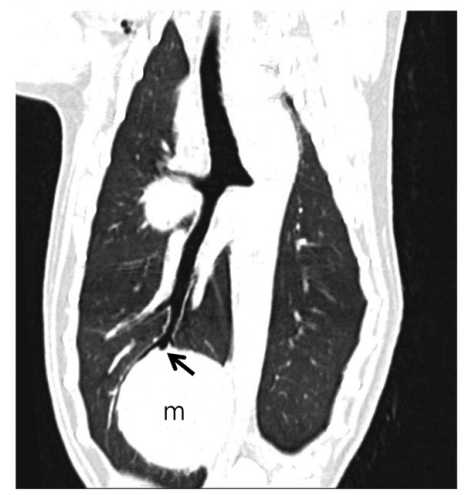

Fig. 2. CT images of the dog. In pre-contrast $\mathrm{CT}$ images (A, D and $\mathrm{G})$ at soft tissue window setting (window level=40, window width=400), all lung nodules (arrows) showed heterogeneous density consisting of hypoattenuating center (c). In precontrast $\mathrm{CT}$ images $(\mathrm{B}$ and $\mathrm{E}$ ) at lung window setting (window level $=-600$, window width $=1,200$ ), the pleural indentations (arrows) were found in the right cranial and left caudal nodules (m). On post contrast CT images (C, F and H), partial contrast enhancement (arrows) occurred from peripheral parenchyma of nodules (m) (window level $=40$, window width $=400$ ). The size of the right caudal nodule, the largest one, was $3.2 \times 2.7 \mathrm{~cm}$ in diameter. In reconstructed dorsal planes, note the obstruction of right caudal bronchus (arrow) by the right caudal nodule $(\mathrm{m})$ in (I) and the cranial deviation of right cranial bronchus (arrow) by the right cranial nodule (m) in (J). The left side of the image is the right side of the dog. 


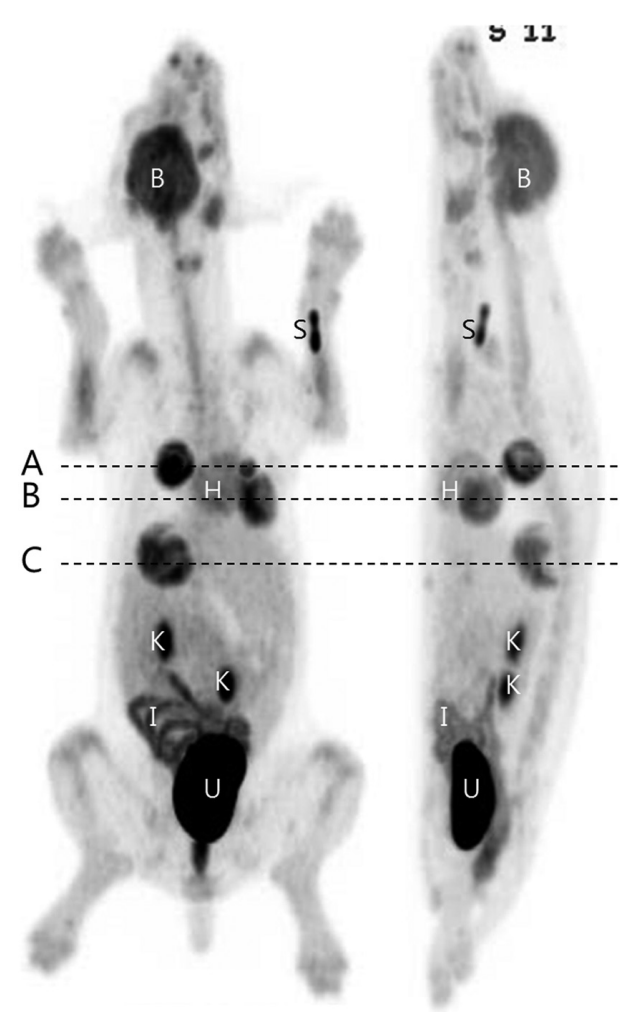

Fig. 3. Positron emission tomography (PET) images using 18F-fluorodeoxyglucose (FDG). Maximum intensity projection (MIP) view showed four hypermetabolic lesions in both lungs, and no significant FDG uptake was found from other regions (including mammary gland), except for several physiologic uptakes (esp. brain, heart, kidneys, intestine, urinary bladder and so on). $\mathrm{B}=$ brain, $\mathrm{H}=$ heart, $\mathrm{K}=$ kidney, $\mathrm{I}=$ intestine, $\mathrm{U}=$ urinary bladder, $\mathrm{S}=$ injection site. The left side of the image is the right side of the dog.

with primary lung tumors $[15,16]$. In a previous study of 15 dogs with primary lung tumors, solitary and multiple masses were reported with frequencies of $87 \%$ and $13 \%$, respectively [15]. In a much larger study of 210 dogs with primary pulmonary tumors, the incidence of multiple masses was $37.1 \%$ [16]. Therefore, discrimination between primary and metastatic tumors is not possible based solely on the number of the masses.

A dog with pulmonary metastasis is not a candidate for surgery, so palliative treatment is usually applied. On the other hand, in primary pulmonary tumors, surgery should be considered for removing all lesions, regardless of the number of pulmonary nodules or masses [21]. Therefore, in cases with multiple pulmonary masses, differentiation of the primary pulmonary tumor and metastatic tumors is very important for determining therapeutic options and for predicting the patient's prognosis.

In the generality of cases, multiple pulmonary masses are considered as metastasis, if there is a solitary mass in other viscera. Anatomic diagnostic imaging modalities, especially those with higher spatial resolution, such as CT, can be helpful to determine smaller and uncertain lesions.

CT specific features, especially, of the pulmonary tumor, such as CT bronchus sign, pleural indentation, speculation, ground glass opacity and pseudocavitation, are used for the purpose of differentiating between benign and malignant tumors or determining the surgical plan in humans [1]. CT bronchus sign is a CT finding that is used to evaluate the relationship between a pulmonary mass, and the adjacent bronchus. The bronchus can be affected by a pulmonary mass through obstruction, displacement, compression or narrowing. In this case, the right cranial bronchus was displaced cranially by the pulmonary mass, and the right caudal bronchus was abruptly obstructed by the mass. CT bronchus sign was not identified in the left bronchus around the pulmonary masses. A pleural indentation, a term used when a part of the pulmonary parenchyma is pulled into the mass, was also found in this case. The other human CT features of pulmonary tumors were not identified in this dog, though all CT features are not currently applied in veterinary medicine.

Unknown primary tumor is the diagnosis of a tumor based on metastatic lesions where the primary tumor is not clearly identified. Unknown primary tumor has been reported to be diagnosed for $5-10 \%$ of human patients in which various diagnostic methods have been used [6]. Therefore, extensive investigation using more sensitive diagnostic methods is required. There are certain limits to detecting metastasis in anatomic diagnostic imaging, because these modalities should accompany an increased size or change of the shape of the lesion. PET-CT, one of the typical functional imaging tools for tumors, can detect a tumor on the basis of increased tumor metabolism and physiologic uptake of FDG, even before the development of an anatomic change of the lesion [9, 18]. In humans, PET-CT is recommended as a standard work-up in patients when a tumor is suspected on conventional imaging to identify characteristics of the primary tumor and regional or distant metastasis. In this dog, the pulmonary masses were strongly suspected to be primary pulmonary tumors, by excluding the other primary lesions and metastasis in pulmonary parenchyma. PET-CT results can be classified by the presence or absence of increased FDG uptake and can also be analyzed quantitatively, such as through tumor-to-background ratios [20]. Increased FDG uptake, from 3.8 to 6.4 , was found from each nodule in this dog. In a study about the physiologic uptake of FDG in dogs, the maximum value of SUV in lungs was about 0.90 , which was the lowest uptake in the whole body [11]. Although there is no report about SUV in primary or metastatic lung tumors in dogs, a previous human study investigated the role of FDG PET in the differentiation between lung metastasis and multiple primary lung tumors [5], because the FDG uptake reflects metabolic activity, depending on the tumor types. Based on the SUV of pulmonary nodules in this case, there is possibility that all pulmonary nodules were identical tumors showing similar metabolic activity and one of them was the primary tumor and the others were metastasis. Also, the degree of FDG uptake provides important information useful in predicting the patient's prognosis [17], even though 

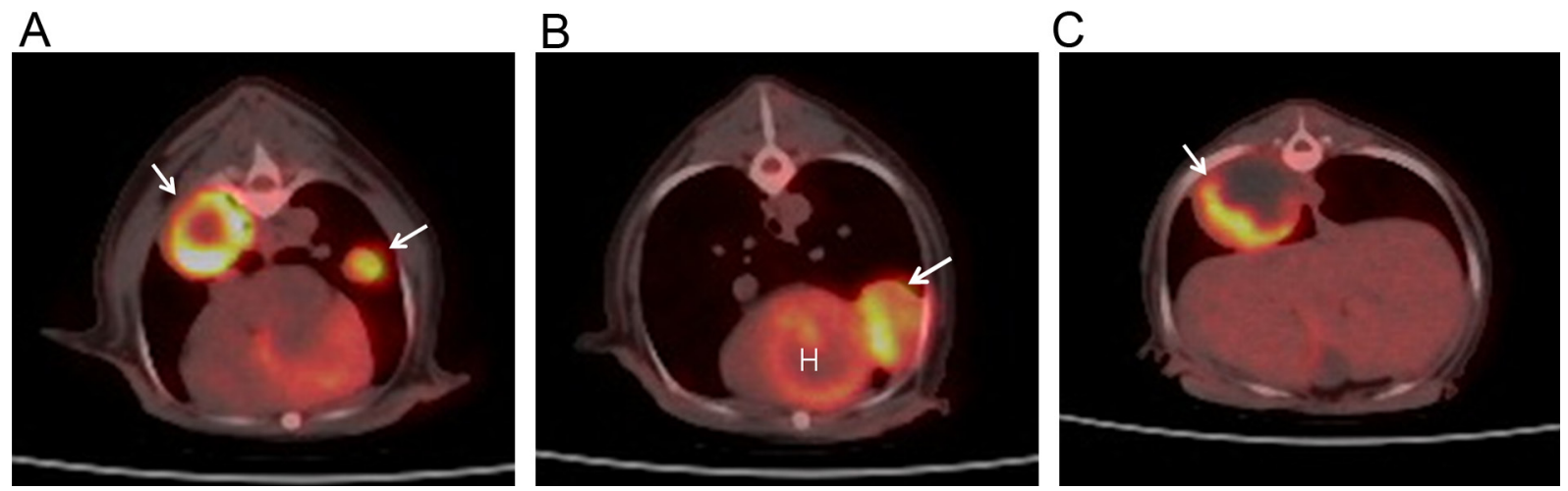

Fig. 4. Axial view of positron emission tomography and computed tomography (PET-CT) fusion images using 18F-fluorodeoxyglucose (FDG). Each image corresponds to the dashed line of Fig. 3 in order from top to bottom. It shows the increased FDG uptake mainly from the peripheral region of each nodule (arrows), which probably resulted from central necrosis $(\mathrm{A}-\mathrm{C}) . \mathrm{H}=$ heart, $\mathrm{L}=$ liver. The left side of the image is the right side of the dog.

TNM staging is used as a reliable prognostic factor in patients with tumors. For example, in clinical stage IA lung adenocarcinoma with high FDG uptake, lymph node metastasis in people more frequently occurred than did low FDG uptake [17]. The criterion for FDG uptake, SUV 3.3, was proposed to be a poor prognosis in humans [17]. In a similar study, a significant correlation was identified between the FDG uptake and the aggressiveness of the adenocarcinoma. That is, the mean SUV in aggressive pulmonary adenocarcinoma $(4.36 \pm 1.94)$ was reported significantly higher than that of non-aggressive ones $(1.53 \pm 0.88)$ [8]. In this dog, the maximum value of SUV would have been considered to be high taking into account the same criterion in human. However, circumstantial assessment of the pulmonary nodule on the basis of the SUV value was impossible, because, to authors' knowledge, there has been no previous study related to FDG PET in canine adenocarcinoma.

Primary pulmonary tumor tends to develop in the peripheral regions in the caudal lung lobe $[12,18]$. Thus, transthoracic fine needle aspiration or biopsy can be considered in pulmonary adenocarcinoma. A transthoracic biopsy under ultrasonography was performed in this case. However, pulmonary adenocarcionoma could not be confirmed histopathologically due to the extent of necrosis. Extensive necrosis was considered as the cause of leukocytosis.

This study described the diagnostic features of radiographs, ultrasonography, CT and PET-CT of primary adenocarcinoma in a dog. In this case, the primary pulmonary tumor was tentatively diagnosed with CT and PET-CT by excluding an undetected primary tumor, and pulmonary nodules could be determined to be malignancies based on the SUV of PET-CT. Although this report is a single case of pulmonary adenocarcinoma in which CT and PET-CT examinations were applied, the procedure of this case can be used as fundamental information for diagnosis of canines with pulmonary adenocarcinoma.

ACKNOWLEDGMENTS. The animal experiment in this study was supported in part by the Animal Medical Institute of Chonnam National University. We thank Myeong-Jun Kim and Sang-Min Han for technical assistance during the PET-CT.

\section{REFERENCES}

1. Akata, S., Fukushima, A., Kakizaki, D., Abe, K. and Amino, S. 1995. CT scanning of bronchioloalveolar carcinoma: specific apperarances. Lung Cancer 12: 221-230. [Medline] [CrossRef]

2. Ballegeer, E. A., Hollinge, C. and Kunst, C. M. 2013. Imaging diagnosis-Multicentric lymphoma of granular lymphocytes imaging with FDG PET/CT in a dog. Vet. Radiol. Ultrasound 54: 75-80. [Medline] [CrossRef]

3. Brodey, R. S. and Craig, P. H. 1965. Primary pulmonary neoplasms in the dog: a review of 29 cases. J. Am. Vet. Med. Assoc. 147: 1628-1643. [Medline]

4. Conti, M. B., Marchesi, M. C., Angeli, G., Lepri, E., Marinetti, C. and Rueca, F. 2010. A case of primary papillary disseminated adenocarcinoma of canine lung. Vet. Res. Commun. 34: S111-115. [Medline] [CrossRef]

5. Dijkman, B. G., Schuurbiers, A. C. J., Vriens, D., LooijenSalamon, M., Bussink, J., Timmer-Bonte, J. N. H., Snoeren, M. M., Oyen, W. J. G., Heijden, H. F. M. and Geus-Oei, L. F. 2010. The role of (18)F-FDG PET in the differentiation between lung metastases and synchronous second primary lung tumours. Eur. J. Nucl. Med. Mol. Imaging 37: 2037-2047. [Medline] [CrossRef]

6. Hainsworth, J. D. and Greco, F. A. 1993. Poorly differentiated carcinoma and poorly differentiated adenocarcinoma of unknown primary tumor site. Semin. Oncol. 20: 279-286. [Medline]

7. Hansen, A. E., McEvoy, F., Engelholm, S. A., Law, I. and Kristensen, A. T. 2011. FED PET/CT imaging in canine cancer patients. Vet. Radiol. Ultrasound 52: 201-206. [CrossRef]

8. Higashi, K., Ueda, Y., Ayabe, K., Sakurai, A., Seki, H., Nambu, Y., Oguchi, M., Shikata, H., Taki, S., Tonami, H., Katsuda, S. and Yamamoto, I. 2000. FDG PET in the evaluation of the aggressiveness of pulmonary adenocarcinoma: correlation with histopathological features. Nucl. Med. Commun. 21: 707-714. [Medline] [CrossRef] 
9. Kole, A. C., Nieweg, O. E., Pruim, J., Hoekstra, H. J., Koops, H. S., Roodenburg, J. L., Vaalburg, W. and Vermey, A. 1998. Detection of unknown occult primary tumors using positron emission tomography. Cancer 82: 1160-1166. [Medline] [CrossRef]

10. LeBlanc, A. K., Miller, A. N., Galyon, G. D., Moyers, T. D., Long, M. J., Stuckey, A. C., Wall, J. S. and Morandi, F. 2012. Preliminary evaluation of serial ${ }^{18} \mathrm{FDG}-\mathrm{PET} / \mathrm{CT}$ to assess response to Toceranib Phosphate therapy in canine cancer. Vet. Radiol. Ultrasound 53: 348-357.

11. Lee, M. S., Lee, A. R., Jung, M. A., Lee, I. H., Choi, J. H., Chung, H. W., Jeong, S. W., Nahm, S. S. and Eom, K. D. 2010. Characterization of physiologic ${ }^{18} \mathrm{~F}-\mathrm{FDG}$ uptake with PET-CT in dogs. Vet. Radiol. Ultrasound 51: 670-673. [Medline] [CrossRef]

12. Lee, Y., Chung, J. H., Kim, S. E., Kim, T. J. and Lee, K. M. 2014. Adenosquamous Carcinoma of the Lung: CT, FDG PET, and Clinicopathologic Findings. Clin. Nucl. Med. 39: 107-112. [Medline]

13. Marolf, A. J., Gibbons, D. S., Podell, B. K. and Park, R. D. 2011. Computed tomographic appearance of primary lung tumors in dogs. Vet. Radiol. Ultrasound 52: 168-172. [Medline] [CrossRef]

14. McNiel, E. A., Ogilvie, G. K., Powers, B. E., Hutchison, J. M., Salman, M. D. and Withrow, S. J. 1997. Evaluation of prognostic factors for dogs with primary lung tumors: 67 cases (1985-1992). J. Am. Vet. Med. Assoc. 211: 1422-1427. [Medline]

15. Mehlhaff, C. J. and Mooney, S. 1985. Primary pulmonary neoplasia in the dog and cat. Vet. Clin. North Am. Small Anim. Pract. 15: 1061-1067. [Medline]
16. Ogilvie, G. K., Haschek, W. M., Withrow, S. J., Richardson, R. C., Harvey, H. J., Henderson, R. A., Fowler, J. D., Norris, A. M., Tomlinson, J. and McCaw, D. 1989. Classification of primary lung tumors in dogs: 210 cases (1975-1985). J. Am. Vet. Med. Assoc. 195: 106-108. [Medline]

17. Ohtsuka, T., Nomori, H., Watanabe, K., Kaji, M., Naruke, T., Suemasu, K. and Uno, K. 2006. Prognostic significance of [(18) F] fluorodeoxyglucose uptake on positron emission tomography in patients with pathologic stage I lung adenocarcinoma. Cancer 107: 2468-2473. [Medline] [CrossRef]

18. Paoloni, M. C., Adams, W. M., Dubielzig, R. R., Kurzman, I., Vail, D. M. and Hardie, R. J. 2006. Comparison of results of computed tomography and radiography with histopathologic findings in tracheobronchial lymph nodes in dogs with primary lung tumors: 14 cases (1999-2002). J. Am. Vet. Med. Assoc. 228: 1718-1722. [Medline] [CrossRef]

19. Review, L. 1973. Radiographic recognition of primary and metastatic pulmonary neoplasms of dogs and cats. Vet. Radiol. Ultrasound 15: 3-24.

20. Sazon, D. A., Santiago, S. M., Soo Hoo, G. W., Khonsary, A., Brown, C., Mandelkern, M., Blahd, W. and Willians, A. J. 1996. Fluorodeoxyglucose-positron emission tomography in the detection and staging of lung cancer. Am. J. Respir. Crit. Care Med. 153: 417-421. [Medline] [CrossRef]

21. Trousse, D., Barlesi, F., Loundou, A., Tasei, A. M., Doddoli, C., Giudicelli, R., Astoul, P., Fuentes, P. and Thomas, P. 2007. Synchronous multiple primary lung cancer: an increasing clinical occurrence requiring multidisciplinary management. J. Thorac. Cardiovasc. Surg. 133: 1193-1200. [Medline] [CrossRef] 